\title{
La epidemiología de los homicidios en Cali, 1993-1998: seis años de un modelo poblacional
}

\author{
Alberto Concha-Eastman, ${ }^{1}$ Victoria E. Espitia, ${ }^{2}$ Rafael Espinosa ${ }^{2}$ \\ y Rodrigo Guerrero ${ }^{3}$
}

RESUMEN Objetivos. Mostrar la utilidad de un modelo eficaz y oportuno de información y llamar la atención acerca de la gravedad del problema de los homicidios, así como demostrar la necesidad de mantener este tipo de modelo y de sostener proyectos preventivos integrales, como el Programa Desarrollo, Seguridad y Paz (DESEPAZ). En el período de 1993 a 1998, en Cali, Colombia hubo 11457 homicidios que fueron registrados mediante un modelo de vigilancia epidemiológica inscrito en DESEPAZ, de la Alcaldía de Cali.

Métodos. A partir de enero de 1993, un grupo de trabajo organizado por el DESEPAZ revisó y homogeneizó las variables que diversas instituciones recopilaron sobre las víctimas, los victimarios y las características de los hechos, y produjo un boletín semanal de resumen para el alcalde y las demás autoridades municipales.

Resultados. Las tasas de homicidios aumentaron de 23 a 124 por 100000 habitantes en el período de 1983-1994. Posteriormente disminuyeron en 1995, 1996 y 1997 a 112, 102 y 86,1 por 100 000, respectivamente, y en 1998 ascendieron ligeramente hasta 88 por 100000 habitantes. Aunque han sido víctimas de homicidios personas de todas las edades, hasta las menores de 5 años, los hombres entre los 20 y 34 años fueron los más afectados. La razón de hombre a mujer ha oscilado entre 14,3:1 y 19,2:1. Los estratos económicos bajos son los más afectados en número, proporción y tasa, aunque los del estrato alto han presentado tasas que llegan hasta 160 por 100000 habitantes. En más del 80\% de los homicidios se utilizó un arma de fuego y el siniestro ocurrió con mayor frecuencia en horas de la noche y durante los fines de semana. En pocos casos (entre 8 y 21\%) hubo un sindicado por el hecho. El análisis con dos variables mostró una asociación positiva con el consumo de alcohol por la víctima, y también con el uso de armas de fuego por el victimario (OR: 3,1; IC95\%: 2,6 a 3,6). En los casos que ocurrieron durante una riña personal o de grupo se observó su asociación con el uso de un arma cortopunzante y con el consumo de bebidas alcohólicas por parte de la víctima (OR: 1,9; IC95\%: 1,4-2,6).

Conclusiones. Se presenta un mapa de la distribución de los homicidios por barrio y se comentan los beneficios del modelo de vigilancia de base poblacional, en especial su valor para identificar los factores de riesgo y las medidas para prevenir y controlar este tipo de violencia.

Palabras clave Violencia, homicidio, Colombia.

Programa de Enfermedades no Transmisibles, Organización Panamericana de la Salud, $52523 \mathrm{rd} \mathrm{St}$, NW, Washington, D.C., 20037, EE. UU. Correo electrónico: conchaal@paho.org

2 Centro de Investigaciones Salud y Violencia (CISALVA), Universidad del Valle, Cali, Colombia.

3 Fundación Carvajal, Cali, Colombia.
Según datos de la Secretaría Municipal de Salud, basados en los Certificados de Defunción expedidos por el Instituto de Medicina Legal y Ciencias Forenses de Colombia (IMLCF),
Regional Sur, la tasa de homicidios en Cali se incrementó de 23 a 93 por 100000 habitantes en el período de 1983 a 1992. Los homicidios llegaron a ser la primera causa de mortalidad 
general para todas las edades, superando a las enfermedades isquémicas cardiovasculares, cuyas tasas por 100000 habitantes oscilaban entre 52 y 54 entre 1992 y 1995 (1). Por otra parte, los informes de otras instituciones, como la Policía y la Fiscalía, no coincidían ni con los del IMLCF ni entre sí. Las diferencias en sus datos eran notorias y explicables por las distintas formas de registro de cada instancia.

En enero de 1993 se conformó un grupo de trabajo compuesto por representantes de las diversas fuentes de datos, es decir, la Fiscalía, la Policía, la Secretaría Municipal de Salud Pública y la Oficina de Derechos Humanos. Este grupo ha sido una pieza fundamental del Proyecto Epidemiología de la Violencia y a su vez componente del Programa Desarrollo, Seguridad y Paz (DESEPAZ) de la Alcaldía de Cali (25), y su tarea ha sido unificar, analizar y diseminar información sobre los homicidios y otras muertes por causas externas. Una epidemióloga de tiempo completo asumió la coordinación del grupo de trabajo. A partir de entonces, las fuentes, antes discordantes, han unificado sus datos y mantenido la coherencia requerida. De esta manera se ha logrado establecer una base de datos confiable, que aún funciona, sobre muertes por causas externas en Cali (6).

El presente trabajo utiliza el registro del DESEPAZ para analizar la información sobre homicidios y en él se usan como variables las características sociodemográficas de las víctimas, los victimarios y los hechos. Sus objetivos son mostrar la utilidad de un modelo de información eficaz y oportuno, resaltar la gravedad de la situación, y demostrar la necesidad de mantener este tipo de modelo y proyectos preventivos integrales como el DESEPAZ. Se examinan todos los homicidios que ocurrieron cada año y el número total durante los seis años transcurridos de 1993 a 1998.

\section{MATERIALES Y MÉTODOS}

La población de estudio estuvo conformada por todos los homicidios que ocurrieron en Cali en el período entre el 1 de enero de 1993 y el 31 de diciembre de 1998 y que fueron registrados en el contexto del Proyecto Epidemiología de la Violencia, del Programa DESEPAZ, que funciona en la Alcaldía de Cali desde junio de 1992.

El registro abarca todos los casos que hubo en el perímetro del municipio, tanto en el área urbana como rural, independientemente de si las víctimas eran residentes locales o no. En los casos de heridos fallecidos con posterioridad a la fecha de la lesión, se registra la fecha en que se produjo el hecho. Del registro llevado por el DESEPAZ se procura excluir aquellos casos de homicidio en que la lesión inicial que llevó a la muerte ocurrió fuera de Cali, y en estos casos, el homicidio se asignó al municipio de origen.

Con el fin de conseguir que todos los homicidios fuesen incluidos, se organizó un grupo de trabajo con representantes de las dependencias oficiales que, por sus funciones, recogen la información pertinente: la Policía, la Oficina de Inteligencia Judicial de la Policía, la Fiscalía, el Instituto de Medicina Legal, la Secretaría de Salud Municipal y la Personería Municipal para la Defensa de los Derechos Humanos. Durante las reuniones semanales de este grupo de trabajo se examinaban todos los casos, se asignaba la causa de muerte, se resolvían incongruencias $y$, si era necesario, se procedía a buscar información adicional para aclarar dudas.

Al inicio del Proyecto, cada una de las variables fue definida de manera operativa mediante un acuerdo entre las instituciones comprometidas. Las variables relativas a la víctima fueron su identidad, edad, sexo, dirección, barrio y el estrato socioeconómico de la residencia; grado de alcoholemia y pertenencia a algún grupo especial de riesgo (dentro de esta categoría se encuentran los mendigos, recolectores de basura, trabajadores sexuales callejeros, travestis callejeros, trabajadores de la construcción y vigilantes). Las variables relativas al victimario fueron su identidad y su pertenencia a grupos tales como pandillas juveniles o fuerzas del estado. Del siniestro se regis- traron el día y la hora, la comuna y el barrio, el tipo de arma utilizada y el motivo aparente.

El grado de alcoholemia fue evaluado por el Instituto de Medicina Legal de Cali, que define como positivas las concentraciones superiores a 50 $\mathrm{mg} / \mathrm{dL}$ de alcohol en sangre. La clasificación socioeconómica de la víctima se hizo de acuerdo con los criterios del Departamento de Planeación Municipal, basados en el barrio de residencia y las características de la vivienda. Esta clasificación asigna al estrato 1 a los más pobres y al 6 a los más ricos. Para las comunas se utilizó la división político-administrativa de la ciudad, conformada por 20 comunas que agrupan barrios vecinos con algún grado de similitud socioeconómica.

La información se introdujo digitalmente en un programa de captura y salida de datos basado en DBase y Clipper. En los análisis realizados periódicamente se describía cada una de las variables recolectadas y su interacción mediante gráficas y cuadros, y se calculaban las tasas de homicidio por barrio, comuna, ciudad y estrato socioeconómico de las víctimas. El sitio de cada caso se ubicaba dentro de las coordenadas de la ciudad mediante un sistema de información geográfica, y con esta información se producían los diversos mapas.

Las frecuencias, correlaciones $y$ pruebas estadísticas se realizaron con el sistema Epi-Info 6.04. Para calcular las tasas acumuladas se utilizaron los promedios de homicidios y los datos oficiales de población de Cali, según la Oficina Municipal de Planeación. El mapa se tomó del Sistema de Información Geográfica.

\section{RESULTADOS}

\section{Generales}

En los seis años entre 1993 y 1998 se registraron 11457 homicidios en la ciudad de Cali, y en 95\% de ellos estaban involucrados residentes de la ciudad. El mayor número de homicidios tuvo lugar en 1994, con un total de 2239 personas asesinadas. A partir 
de 1995 se observó un descenso progresivo hasta alcanzar 1631 asesinatos en 1997, aunque en 1998 se registró un aumento de 3,9\% (64 casos más) con respecto al año anterior. Las tasas reflejan también el cambio detectado (cuadro 1, figura 1).

Los hombres fueron víctimas de homicidios en una proporción mayor que las mujeres. La razón de hombre a mujer para todos los grupos de edad fue de 14,7:1 durante los 6 años. La razón más alta $(19,5: 1)$ se observó en el grupo de 20 a 24 años, seguida de 17,2:1 en el grupo de 40 a 44 años. Las tasas reflejaron un riesgo mucho mayor para los hombres en todos los años y grupos de edad (cuadro 2).

El análisis por sexo y edad revela que los hombres más afectados fueron los mayores de 15 años y, de ellos, los de los grupos de 20 a 24 años y de 25 a 29 años tuvieron tasas cuatro veces superiores a la tasa general. Sin embargo, debe destacarse que en determinados años, en otros grupos de edad también se observaron tasas muy elevadas en los hombres, como los de 35 a 39 años en 1994 (450/ $100000)$ y los de 30 a 34 años en 1996 $(357 / 100$ 000). Las mujeres presentaron tasas mucho más bajas que los hombres en todos los grupos de edad y en todos los años del estudio. Se observó un incremento a partir de los 15 años, pero las tasas mayores en los distintos grupos de edad variaron de un año a otro.

La figura 2 presenta las tasas específicas por edad y sexo para cada uno de los 6 años del estudio. Puede observarse que el pico de las tasas masculinas se redujo a partir de 1994 y, en general, todas las tasas específicas disminuyeron a partir de ese año. Los picos de las tasas masculinas entre los

CUADRO 1. Cambios en el número y tasas de homicidios. Cali, 1993-1998

\begin{tabular}{lcccc}
\hline Año & No. & Cambio porcentual & Tasa (por 100 000) & Cambio en la tasa \\
\hline 1993 & 1831 & $+13,7^{\mathrm{a}}$ & 104 & $+11^{\mathrm{b}}$ \\
1994 & 2239 & $+22,3$ & 124 & +24 \\
1995 & 2063 & $-7,9$ & 112 & -12 \\
1996 & 1990 & $-3,5$ & 106 & $-5,3$ \\
1997 & 1631 & $-18,0$ & 86 & -16 \\
1998 & 1695 & $+3,9$ & 89 & $+3,5$ \\
Total & 11449 & - & - & - \\
\hline
\end{tabular}

Fuente: Secretaría de Salud de Cali.

a Casos en 1992: 1610.

b Tasa en 1992: 93 por 100000

\section{FIGURA 1. Tasas (por 100000 habitantes) de homicidos en Cali, 1983-1998}

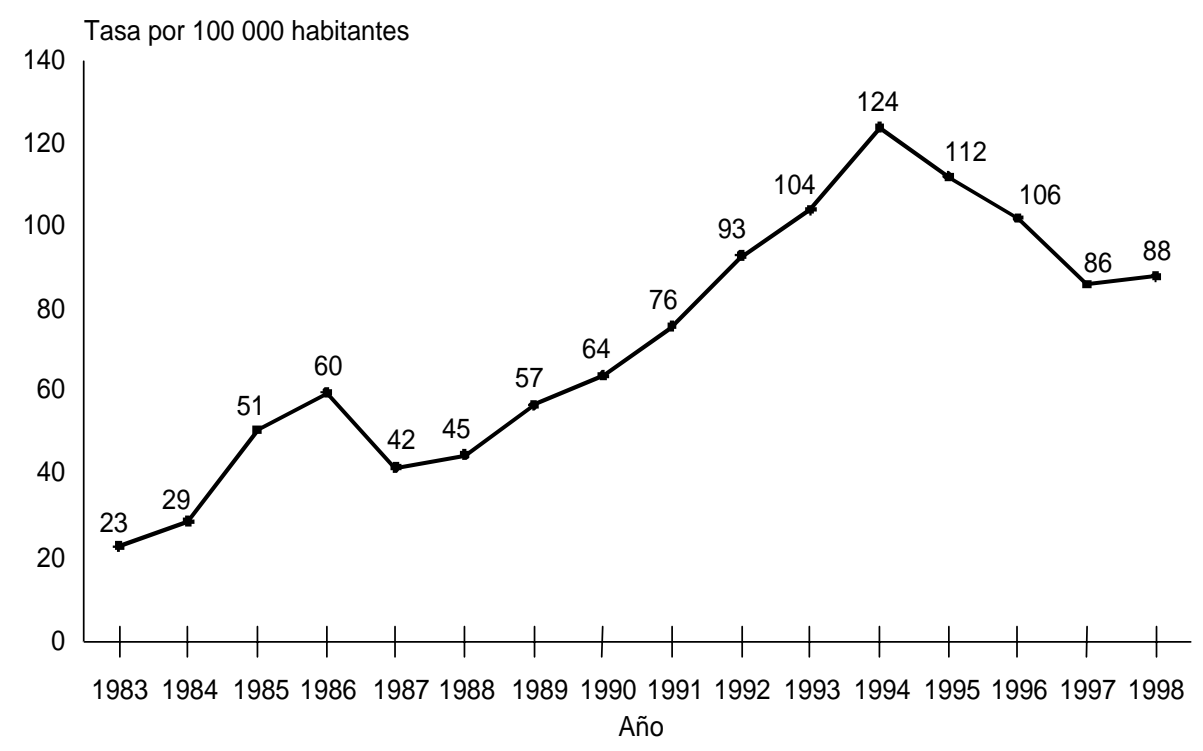

Fuentes: Secretaría de Salud Pública Municipal (1983-1992); Fiscalía, CTI, Personería, Policía Metropolitana, Instituto de Medicina Legal, Salud Pública y Centro de Diagnóstico Automotre (1993-1998).

años 1995 y 1996 se desplazaron hacia el grupo de edad de 20-24 años. En 1997 las tasas fueron similares en los grupos de edad de 20-24 y 25-29, mientras que en 1998 la curva se desplazó hacia el grupo de 25-29 años.
Llama la atención que 44 niños menores de 5 años y 23 niños de 5 a 9 años de edad fueron asesinados en este período, por lo general mientras sus padres fueron baleados o cayeron en fuego cruzado.

CUADRO 2. Homicidios y tasas (por 100000 habitantes) según sexo. Cali, 1993-1998

\begin{tabular}{|c|c|c|c|c|c|c|c|c|c|c|c|c|c|c|}
\hline \multirow[b]{2}{*}{ Sexo } & \multicolumn{2}{|c|}{1993} & \multicolumn{2}{|c|}{1994} & \multicolumn{2}{|c|}{1995} & \multicolumn{2}{|c|}{1996} & \multicolumn{2}{|c|}{1997} & \multicolumn{2}{|c|}{1998} & \multicolumn{2}{|c|}{ Total } \\
\hline & No. & $\overline{\text { Tasa }}$ & No. & Tasa & No. & Tasa & No. & Tasa & No. & Tasa & No. & $\overline{\text { Tasa }}$ & No. & Tasa $^{\mathrm{a}}$ \\
\hline Hombres $(\mathrm{H})$ & 1712 & 209 & 2083 & 245 & 1920 & 222 & 1878 & 214 & 1518 & 171 & 1611 & 182,5 & 10729 & 204 \\
\hline Razón H/M & 14,4 & 16,1 & 12,7 & 15,3 & 13,4 & 14,8 & 16,8 & 17,3 & 13,6 & 15,5 & 19,2 & 21,9 & 14,7 & 17,0 \\
\hline
\end{tabular}

a Tasa promedio para 6 años. 
FIGURA 2. Tasas (por 100000 habitantes) de homicidos según grupo de edad y sexo. Cali, 1993-1998

1993

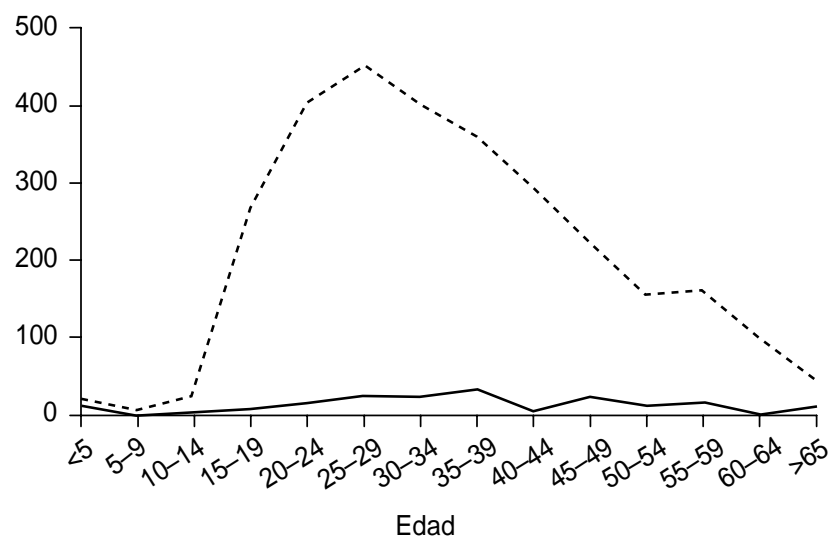

1995

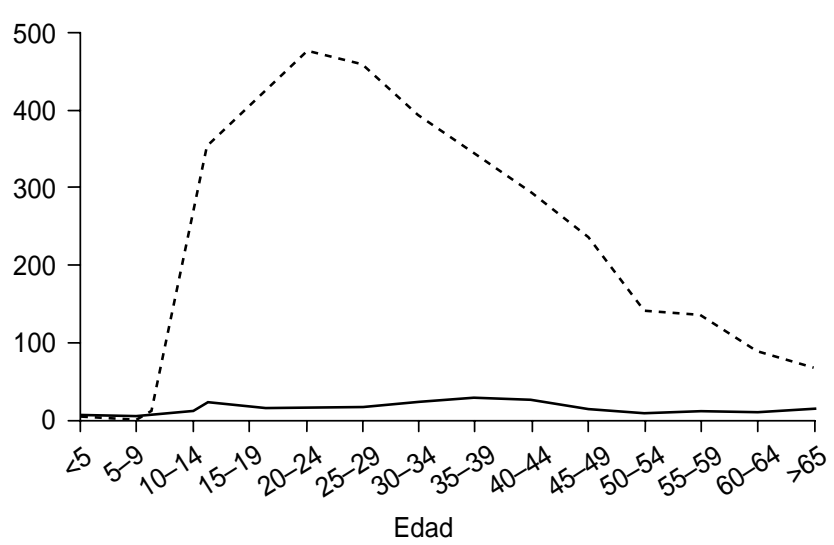

1997

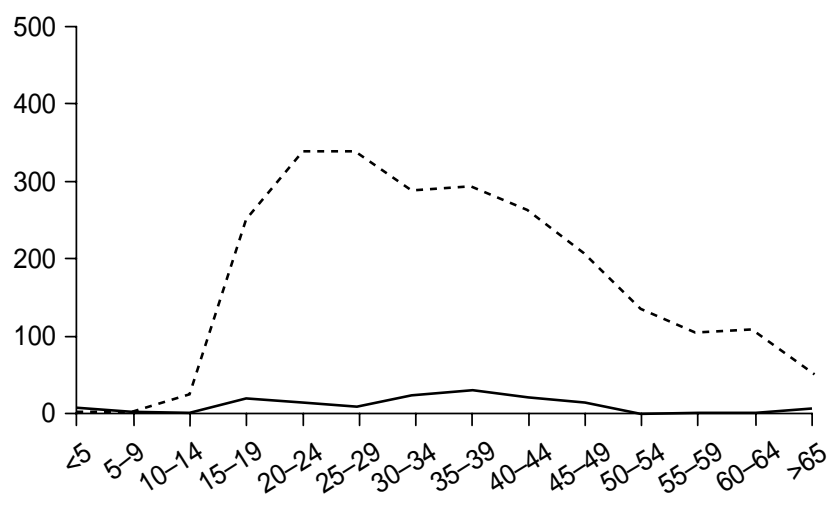

Edad
1994

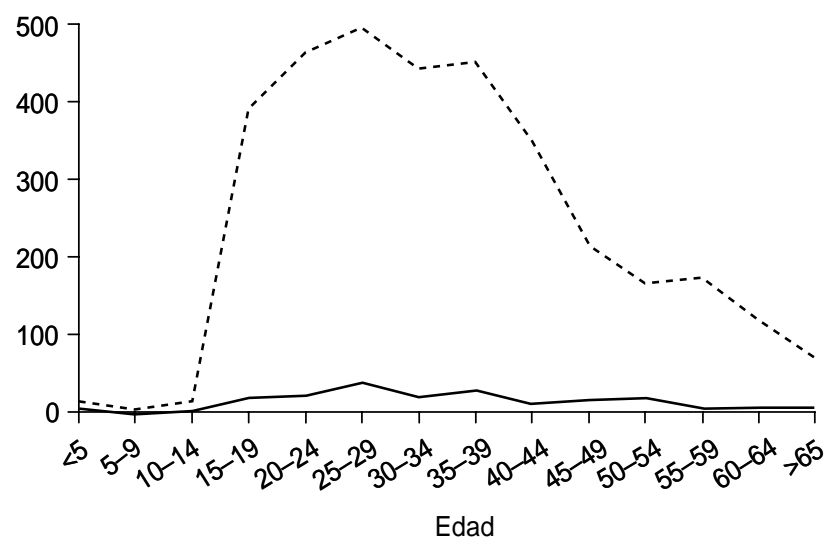

1996

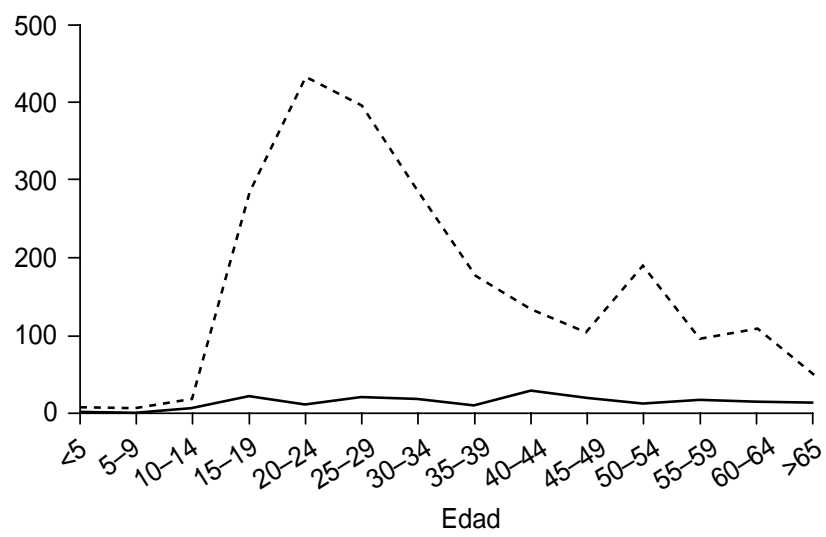

1998

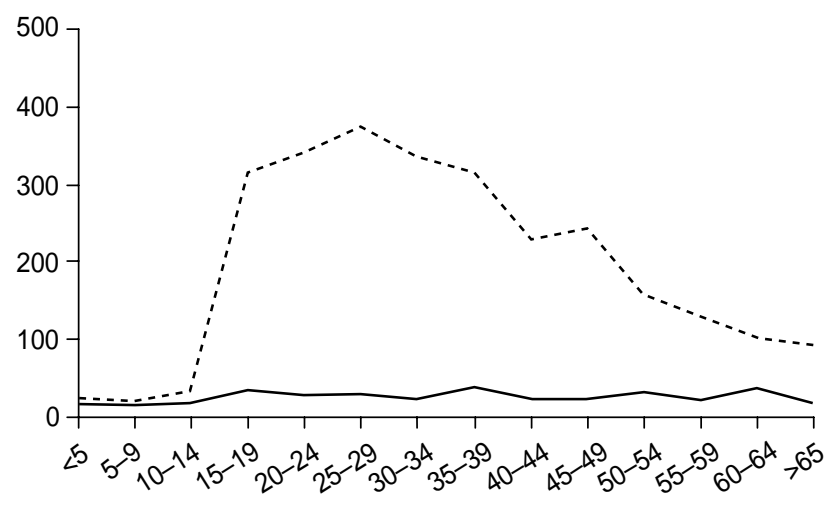

Edad

\section{-...- Hombres $\quad$ Mujeres}

Fuentes: Policía Metropolitana, Fiscalía, Personería, Medicina Legal y Centro de Diagnóstico Automotor. 
CUADRO 3. Tasas (por 100000 habitantes) según grupos de edad y sexo. Cali, 1993-1998

\begin{tabular}{|c|c|c|c|c|c|c|c|c|c|c|c|c|}
\hline \multirow[b]{2}{*}{ Edad } & \multicolumn{2}{|c|}{1993} & \multicolumn{2}{|c|}{1994} & \multicolumn{2}{|c|}{1995} & \multicolumn{2}{|c|}{1996} & \multicolumn{2}{|c|}{1997} & \multicolumn{2}{|c|}{1998} \\
\hline & Hombres & Mujeres & Hombres & Mujeres & Hombres & Mujeres & Hombres & Mujeres & Hombres & Mujeres & Hombres & Mujeres \\
\hline$<5$ & 18 & 8 & 12 & 6 & 2 & 3 & 7 & 5 & 2 & 4 & 6 & 0 \\
\hline $5-9$ & 1 & 1 & 1 & 0 & 2 & 2 & 3 & 0 & 1 & 3 & 3 & 0 \\
\hline $10-14$ & 20 & 1 & 13 & 4 & 12 & 10 & 15 & 5 & 21 & 2 & 15 & 2 \\
\hline $15-19$ & 267 & 6 & 389 & 17 & 354 & 23 & 345 & 16 & 250 & 17 & 309 & 15 \\
\hline 20-24 & 402 & 16 & 462 & 23 & 475 & 18 & 420 & 16 & 338 & 13 & 325 & 14 \\
\hline $25-29$ & 450 & 25 & 495 & 37 & 456 & 15 & 326 & 17 & 338 & 9 & 360 & 13 \\
\hline $30-34$ & 400 & 24 & 336 & 23 & 391 & 22 & 357 & 17 & 286 & 23 & 327 & 7 \\
\hline 35-39 & 356 & 33 & 450 & 26 & 343 & 27 & 266 & 11 & 291 & 28 & 306 & 17 \\
\hline $40-44$ & 293 & 5 & 349 & 13 & 292 & 26 & 200 & 18 & 260 & 21 & 213 & 11 \\
\hline $45-49$ & 219 & 22 & 211 & 16 & 236 & 14 & 180 & 13 & 207 & 13 & 229 & 5 \\
\hline $50-54$ & 152 & 12 & 164 & 18 & 138 & 9 & 156 & 10 & 134 & 0 & 140 & 12 \\
\hline $55-59$ & 161 & 16 & 173 & 8 & 135 & 12 & 78 & 13 & 102 & 4 & 115 & 4 \\
\hline $60-64$ & 97 & 0 & 117 & 5 & 86 & 10 & 80 & 11 & 107 & 0 & 85 & 14 \\
\hline$>65$ & 42 & 8 & 68 & 8 & 67 & 13 & 34 & 9 & 49 & 5 & 78 & 0 \\
\hline
\end{tabular}

Fuente: DESEPAZ, Epidemiología de la violencia.

En el cuadro 4 se muestran otras características importantes de las variables registradas. Durante los fines de semana se observó la mayor frecuencia de homicidios (uno de cada cuatro ocurrió en domingo). En horas de la noche se perpetraron dos de cada tres homicidios. Entre 13,0\% y 23,4\% de las víctimas tenían $50 \mathrm{mg} / \mathrm{dL}$ de alcohol en sangre o más. Sin embargo, en un elevado número de casos no se detectó alcoholemia.

El móvil identificado más frecuentemente fue el atraco, seguido de la riña (18,2\% de la cifra acumulada para todo el período), pero la alta proporción de casos sin un móvil conocido impidió caracterizar adecuadamente esta variable.

Se determinó el tipo de victimario en $20 \%$ a $27 \%$ de los casos durante los 6 años abarcados en el estudio. Los sicarios (asesinos de oficio) aparecieron en mayor proporción $(15,0 \%)$, seguidos de los miembros de pandillas juveniles. La categoría "desconocido" fue la más frecuente dentro de esta variable, y su tendencia permaneció estable en los 6 años.

Los estratos socioeconómicos más bajos fueron los más afectados en términos de número, proporción y tasa de homicidios, y los hechos ocurrieron con mayor frecuencia en barrios de escasos recursos. En efecto, 3445 y 3764 personas murieron en barrios de los estratos 1 ó 2 , respectivamente -donde vivía $63,5 \%$ de todos los casos-, con tasas de 175 a 251 por 100000 habitantes para los barrios del estrato 1, y de 136 a 163 por 100000 habitantes para los del estrato 2. Sin embargo, la tasa para los del estrato 6 fue también alta y fluctuó entre 195/100 000 (en 1993) y 143/100 000 (en 1995). La población clasificada en el estrato 4 tuvo las tasas más bajas —entre 16 y 20 por 100000 habitantes- en los 6 años. Si se agrupan los tres estratos inferiores, la proporción de casos aumenta de 82,0\% a 90,0\% entre 1993 y 1998, es decir, que un mínimo de 8 de cada 10 asesinatos se cometieron en los barrios más pobres.

La proporción de muertes según el estrato del lugar donde se produjo el siniestro y donde residía la víctima se mantuvo estable durante los 6 años. La proporción de personas asesinadas en comunas que pertenecían al mismo estrato socioeconómico que su residencia disminuyó en los estratos superiores: estrato 1: $71,3 \%$; 2: $69,7 \%$; 3 : 54,4\%; 4: 29,8\%; 5: 34,1\%; y 6: 24,1\% $\left(\chi^{2}\right.$ de tendencia lineal $=17,6 ; P=0,00003$, tomando como base el estrato 6). Esto puede significar que cuanto más bajo era el estrato, más alta era la probabilidad de que el homicidio ocurriera cerca del lugar de residencia.

En algunas comunas se observaron tasas que duplicaban las de la ciudad. En 1994, por ejemplo, la comuna con la mayor tasa de homicidios tuvo una cifra 2,2 veces más alta que la de Cali. Por otro lado, en 1996 la comuna que tuvo la menor tasa tuvo una cifra 5,6 veces menor que la de esta última ciudad. Debe destacarse, asimismo, que en algunos barrios no hubo ningún homicidio en el período estudiado, tal como se observa en la figura 3 , que muestra esquemáticamente las tasas de homicidios por barrio durante 1996. Como puede observarse, en la mayoría de los barrios las tasas son inferiores al promedio de toda la ciudad. Esto significa que se puede dar por sentada una agregación "social" del fenómeno.

El arma de fuego fue la más utilizada para cometer el crimen y a lo largo del período estudiado su uso aumentó en relación con el de las armas cortopunzantes. En términos absolutos, sin embargo, la tasa de homicidios por arma de fuego disminuyó gradualmente de 98,5 en 1994 a 74,6 en 1998.

Durante los 6 años estudiados, se observó un aumento significativo del número de víctimas y victimarios identificados (víctimas: de 92,2\% a $97,0 \% ; \chi^{2}$ de tendencia lineal $=22,5$, $P<0,00001$; victimarios: de $8,2 \%$ a $21,0 \% ; \chi^{2}$ de tendencia lineal $=78,4$, $P<0,000001)$.

No se observó tendencia estacional alguna en los homicidios. En los 6 años, el número mensual de casos mostró fluctuaciones, aunque en febrero - que tiene menos días y ningún 
CUADRO 4. Características predominantes (\%) de 11457 homicidios. Cali, 1993-1998

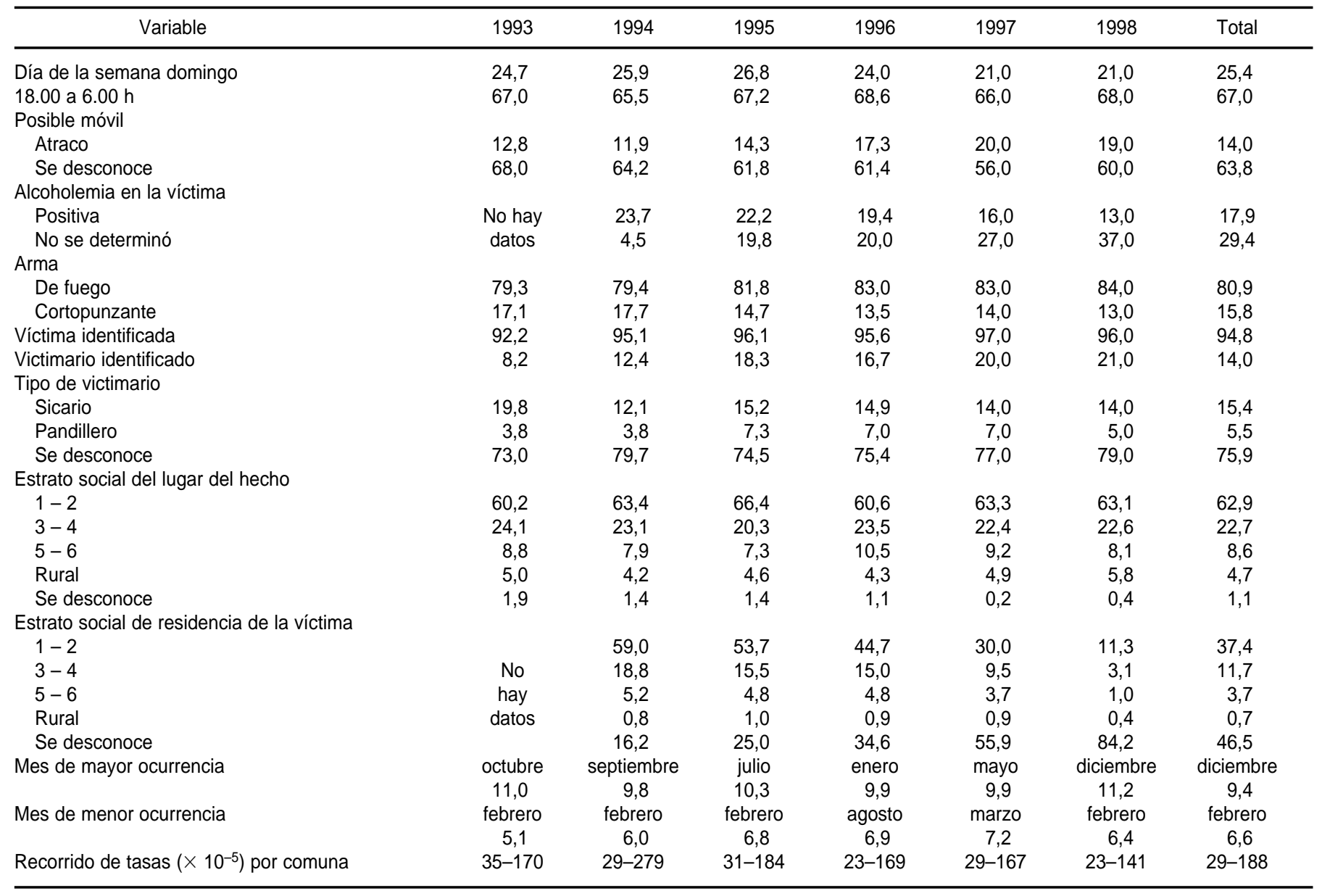

día de fiesta- se registraron menos casos que en los demás meses en 1993, 1994, 1995 y 1998. En diciembre de 1998 se produjo el mayor número de casos (199) y el mayor valor acumulado de todos los 6 años (1 108 muertes; $9,7 \%$ del total). Es posible que esto se relacione con las festividades de Navidad, Feria de Cali y Año Nuevo, en las cuales el consumo de alcohol es mayor. Nótese que hubo poca diferencia entre los porcentajes correspondientes a los meses en que se produjeron más y menos casos — de 3,0 a 5,9-, lo cual corrobora la ausencia de una variación estacional.

Se registraron 1048 homicidios (9,1\% del total) en personas clasificadas dentro de grupos de riesgo especial. De estos, los trabajadores de la construcción fueron las víctimas más frecuentes, con 418 casos $(39,9 \%)$, seguidos por los mendigos, con 176 (16,8\%), los vigilantes $147(14,0 \%)$, los basureros 110 (10,5\%), los drogadictos 150 (14,3\%), los homosexuales $22(2,1 \%)$ y las trabajadoras sexuales $16(1,5 \%)$. La impunidad en este grupo es de casi $100 \%$ y en $98,9 \%$ de estos casos ni siquiera hubo un acusado. En los ocho casos en que hubo algún sindicado, los victimarios fueron clasificados como pandilleros.

\section{Análisis con dos variables}

Se realizaron tantos cruces de variables como la lógica y el tipo de variable lo permitieran. Los datos estadísticos que se muestran y comentan a continuación representan las variables que se consideraron más importantes, ya fuera con asociación estadística significativa o sin ella.
- En los homicidios con arma de fuego, el tipo de arma se asoció con el consumo de bebidas alcohólicas por parte de la víctima (OR: 2,2; IC95\%: 2,0 a 2,4).

- Hubo asociación estadística entre las víctimas de los estratos residenciales 1,2 y 3 y el consumo de bebidas alcohólicas (OR: 2,0; IC95\%: 1,6 a 2,4$)$, pero no entre este y los estratos 4,5 y 6 .

- En los homicidios con arma cortopunzante fue más probable que se sentenciara a alguna persona por el crimen, no así cuando este se cometió con arma de fuego. Este hallazgo se mantiene constante durante todo el período (OR: 2,6; IC95\%: 2,3 a 2,9).

- Cuando el homicidio estaba vinculado con una riña personal, hubo asociación entre el uso de arma cor- 
FIGURA 3. Mapa de la distribución por barrios de las tasas de homicidios cometidos en Cali, 1996

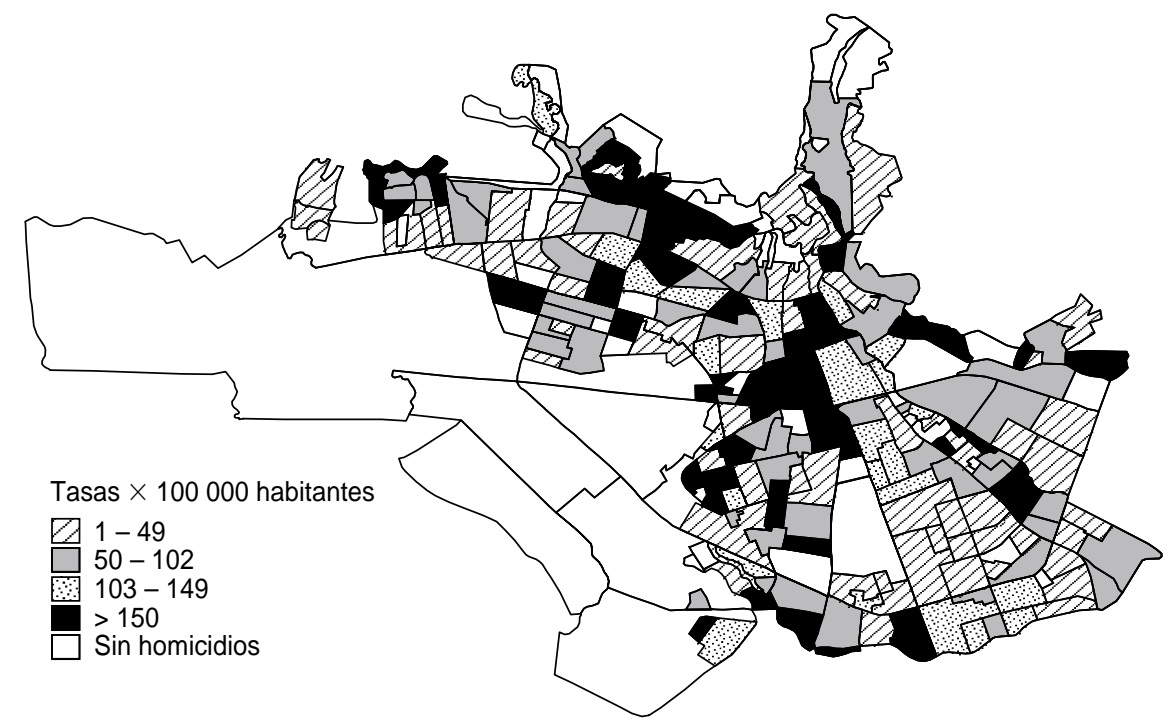

topunzante y el consumo de bebidas alcohólicas (OR: 1,9; IC95\%: 1,4 a 2,6$)$.

- Durante los 6 años se encontró una asociación estadística entre el móvil del atraco y las riñas, por un lado, y la ocurrencia del siniestro en horas diurnas (OR: 1,5; IC95\%: 1,2 a $1,8)$, pero esta asociación fue débil en 1994 (OR: 1,5; IC95\%: 1,05 a 2,2) y no se demostró en 1995 (OR: 1,1; IC95\%: 0,8 a 1,6).

- En el caso de las víctimas del sexo femenino se encontró lo siguiente:

a) Una mayor probabilidad de que se sentenciara a una persona por el crimen (OR: 1,9; IC95\%: 1,6 a 2,3).

b) Ninguna asociación con el consumo de bebidas alcohólicas por parte de las víctimas (OR: 0,4; IC $95 \%$ : 0,3 a 0,5).

c) Ninguna diferencia en cuanto al tipo de arma (de fuego o cortopunzante) utilizada en el crimen (OR: 1,06; IC95\%: 0,8 a 1,4).

d) Para las mujeres asesinadas de los estratos 4,5 y 6 se encontró una asociación positiva con un victimario identificado, no así en las de los estratos 1, 2 y 3 (OR: 1,4; IC95\%: 1,1 a 2,8). por otras autoridades nacionales (Bogotá y Medellín) y extranjeras (San Pedro Sula, Honduras, y Quito, Ecuador, entre otras).

Es alarmante, por otro lado, que no haya suficiente información acerca de algunas variables para poder sacar conclusiones, particularmente en lo que respecta a la identidad del victimario y el motivo del hecho. La magnitud del problema, reflejada en las altas tasas de homicidio en la ciudad y en todos los estratos socioeconómicos y comunas, no solo justifica la operatividad de este modelo, sino que invita a mantenerlo y mejorarlo. De hecho, las tasas más bajas registradas por estrato socioeconómico (16 por 100000 habitantes para el estrato 4, en 1993) y por comuna (23 en la comuna 5, en 1996) son altas para los estándares internacionales (8). En efecto, las tasas de Cali son superiores a las tasas promedio de toda Colombia (87/100 000 en 1993 y 77/100 000 en 1995 [9]), y a las de Bogotá en 1996 (56,3/100 000), pero inferiores a las de otras ciudades del país, como Medellín, que tuvo en 1996 una tasa de 271 por 100000 habitantes (9).

Las causas de estos crímenes no se han podido determinar debido, especialmente, a la gran impunidad reinante. Se estima que solo $10 \%$ o menos de los homicidios perpetrados en Colombia se castigan. En Cali la situación es similar, pues algunos estudios (10) estiman que en solo de $3 \%$ a $7 \%$ de los homicidios se condena a una persona. Entre las causas conocidas de estos crímenes figuran el narcotráfico, cuya aparición en la ciudad coincide con el incremento de las tasas de homicidios registradas en los años ochenta. Este es el único factor general, como fenómeno social, que surge como una nueva actividad a finales de la década de 1970 y que, dado su alto nivel delictivo y corruptor, tuvo un efecto negativo sobre amplios sectores sociales. El narcotráfico y sus actividades conexas han producido la muerte de personas directamente vinculadas con esa actividad y de otras personas ajenas a ella, especialmente líderes abiertamente opuestos a estas actividades delictivas. También generan muertes de manera indirecta, ya que fomentan la noción 
de que los conflictos personales se resuelven por la vía del aniquilamiento del contrario, y en algunos sectores sociales llevan al surgimiento de pandillas o bandas delictivas a las que se les han imputado muchos de los homicidios. Sin embargo, no se conoce con precisión cuántos de los homicidios son imputables al narcotráfico. Se especula que alrededor de $30 \%$, aunque esta cifra no tiene un fundamento real.

Otros factores asociados con los homicidios y los actos violentos siempre han estado vinculados con la pobreza. Los datos presentados en este artículo parecen apoyar esa hipótesis, ya que el número, la proporción y las tasas de homicidios en los estratos 1 y 2 -a los que pertenece cerca de $45 \%$ de la población caleña $(11,12)$ - han sido elevados durante los 6 años del estudio. Otros datos, sin embargo, contradicen esta hipótesis, ya que hay barrios pobres de la ciudad donde no se han notificado homicidios o donde las tasas correspondientes son muy inferiores al promedio de toda la ciudad y de otros estratos sociales similares (figura 3). Además, en los estratos socioeconómicos más altos, el 5 y el 6, las tasas de homicidios en el mismo período han sido tan altas como las de los estratos inferiores. Un estudio en Chicago, EE.UU., mostró que la pobreza y las diferencias raciales no eran los factores que explicaban las altas tasas de homicidios en esa ciudad, sino más bien el grado de compromiso y organización comunitarios (13). Este tipo de estudios no se han realizado en nuestro medio.

Otros factores que favorecen los actos violentos, como portar armas de fuego o cortopunzantes y el consumo de bebidas alcohólicas, se asocian positivamente con los homicidios en Cali. Este hallazgo, que ha sido constante durante los 6 años de nuestra observación, coincide con el de otros estudios. Rutledge y Messick (14) documentaron una fuerte asociación entre el consumo de alcohol y la muerte por homicidios, accidentes y suicidios. De un total de 12414 muertes traumáticas entre 1986 y 1988, 9151 ocurrieron durante las 24 horas posteriores al trauma (74\%). En $80 \%$ de ellas se de- terminó el grado de alcoholemia y se encontraron niveles positivos — según el parámetro legal de $100 \mathrm{mg} / 100$ $\mathrm{mL}$ - en $42 \%$ de los casos de homicidio, $23,6 \%$ de los suicidios y $34 \%$ de los casos de accidentes. Goodman et al. (15) estudiaron 2300 muertes en el Estado de Oklahoma entre 1978 y 1984, de las cuales 800 (35\%) fueron intencionales (homicidios o suicidios) y 1500 (65\%) no lo fueron. En $90 \%$ de los asesinados se determinó el grado de alcoholemia, con resultados positivos en $52 \%$ de los casos, observándose una mayor prevalencia en los grupos minoritarios, como los latinos y los llamados americanos oriundos (native Americans).

Por otro lado, portar armas ha mostrado una asociación positiva con el riesgo de cometer un asesinato. En Cali, portar un arma de fuego de bajo calibre puede ser o no legal, cosa que depende de decisiones administrativas acordadas, por lo general, entre la Alcaldía, las Fuerzas Militares y la Policía. Desde 1993 se ha establecido con mayor rigor la prohibición de portar armas de fuego, ya sea en sitios públicos, en el vehículo o en cualquier otro lugar. Según los boletines del DESEPAZ, tales decisiones han sido reforzadas durante algunos fines de semana, días de pago de salarios, y en ocasión de festividades colectivas o eventos deportivos internacionales.

Villaveces et al. (16) estudiaron los efectos del desarme de civiles en Cali y Bogotá y concluyeron que durante los días en los que se prohibió y controló el porte de armas de fuego, la tasa de homicidios disminuyó en 13\%. También en Bogotá, el Instituto de Medicina Forense encontró una reducción de los homicidios de 5\% en 1995 y 1996, en comparación con otros dos períodos semestrales iguales de dos años (9). Vassar y Kiser (17), en un estudio retrospectivo de base poblacional, estudiaron 9562 hospitalizaciones por heridas con arma de fuego en California en 1991. Sus hallazgos mostraron que en $74 \%$ de los casos el móvil fue el asalto, lo cual coincide con la mayor frecuencia de este móvil en Cali. Los hombres de raza negra de 15 a 24 años de edad comprendieron $72 \%$ de todas las hospitalizaciones y su tasa de homicidio fue de 439 por 100000 habitantes. Después de sufrir heridas por arma de fuego, la razón del número de egresos hospitalarios al número de muertes fue de $1,8: 1$, lo que refleja la gravedad de los atentados contra la vida cuando se usa este tipo de arma. En 1996, Concha-Eastman y Espinosa (18) establecieron que la razón del número de personas heridas sin fallecer al de personas asesinadas en zonas de Cali en alto riesgo de violencia estuvo entre 3,5:1 y 8,5:1, independientemente del tipo de arma utilizada y de la gravedad de la lesión. Kellerman (19) ha demostrado que portar armas de fuego o tenerlas en la casa incrementa 2,7 veces el riesgo de homicidio.

En el presente estudio se observa un bajo porcentaje de homicidios atribuibles a pandillas juveniles, en oposición a la creencia de muchas personas y a lo difundido por los medios. Otros estudios también han revelado que las pandillas no están implicadas con mayor frecuencia en estos delitos, y que la percepción de la sociedad sobre las actividades de estos grupos merece ser documentada. En Los Ángeles, ciudad donde hay muchas pandillas y donde abunda el tráfico de narcóticos, Meehan y O'Carroll (20) estudiaron la proporción de homicidios en los que podían estar involucrados estos grupos, para lo cual revisaron los archivos de la policía de los homicidios perpetrados entre el $1 .^{\circ}$ de enero de 1986 y el 31 de agosto de 1988, y encontraron una probabilidad relativamente baja de que hubiese homicidios relacionados con el tráfico de narcóticos por pandillas juveniles. Incluso, hallaron menos probabilidades de que los miembros de estas pandillas estuvieran involucrados en los homicidios en que hubo un vínculo comprobado con el tráfico de narcóticos. Vanegas (21) en Cali, y Smutt y Miranda (22) en San Salvador, El Salvador, han realizado estudios etnográficos en los que se ha demostrado que el comportamiento de estas pandillas juveniles puede explicarse mejor como producto de situaciones familiares, conflictos sociales, la ausencia del padre y de conductas 
aprendidas de sus pares, que como producto de una relación con el tráfico de narcóticos $(21,22)$.

Como se destacó anteriormente, las 44 muertes violentas de niños menores de 10 años, 34 de los cuales eran menores de 5 , representan otra faceta de la gravedad de los actos homicidas que amenazan a los pobladores de Cali. Basándose en encuestas de 14 días de niños menores de 16 años con todo tipo de trauma en los pabellones de emergencias de dos grandes hospitales de referencia de Trinidad y Tabago, Kirsch et al. (23) notificaron que $13,9 \%$ fueron consecuencia de actos intencionales, pero solo 3 niños murieron. Singh y Yu revisaron las tendencias y diferencias en la mortalidad de los niños entre 1950 y 1993 en los EE.UU., concluyendo que la patente disminución de enfermedades infecciosas, cáncer, anomalías congénitas y traumatismos no intencionales queda eclipsada por el incremento de las muertes por suicidio y homicidio, que se han duplicado y quizá triplicado desde 1968. Los grupos en riesgo fueron los hombres, las personas de raza negra, los indios, los hawaianos y los puertorriqueños, además de las personas de estratos económicos inferiores (24). En Cali también ha disminuido la mortalidad por enfermedades infecciosas (1), pero estas aún predominan en los estratos económicos más bajos, junto con los homicidios. Los programas de prevención y control del crimen deben tomar en cuenta esta grave situación.

La disminución en Cali del número absoluto de homicidios y de sus respectivas tasas entre 1995 y 1997 indica que es posible controlar y disminuir este tipo de actos. Sin embargo, vistas objetivamente, las cifras continúan siendo extremadamente altas. De Roux (8) resume los datos procedentes de la Organización Panamericana de la Salud, que muestran la evolución de las tasas de homicidio en países como Ecuador, cuya tasa por 100000 habitantes ascendió de 8,0 en 1981 a 11,0 en 1990; Brasil, donde la tasa subió de 16,4 en 1980 a más de 20 en 1990; o Perú, donde la tasa se quintuplicó entre 1986 y 1991. En El Salvador, después de los Acuerdos de Paz de 1992, se ha observado un incremento de los homicidios, especialmente en las zonas urbanas, con tasas entre 140 y 90 por 100000 habitantes entre $1996 \mathrm{y}$ 1999 (25). Estas tasas son mayores que las encontradas en Colombia.

En todos los documentos publicados en la actualidad sobre la delincuencia se reconoce que las acciones para prevenirla deben ser múltiples y multisectoriales, con participación de entidades gubernamentales y no gubernamentales. El Programa DESEPAZ, de la Alcaldía de Cali, así lo considera (2, 3-5). El aporte de la Salud Pública y la Epidemiología se reconocen indispensables para su estudio y prevención. Guerrero (6) y Franco (26) en Colombia, Prothrow-Stith (27) y Rosenberg $(28,29)$ en los EE.UU., así como varios gobiernos, como los de Colombia (30) y México (31), no dudan en afirmar la necesidad de implementar metodologías y políticas para prevenir el crimen de una manera integral, involucrando a los sectores gubernamentales y no gubernamentales que se consideren necesarios para esta tarea, como los Depar- tamentos de Justicia, Salud y Educación y la Policía, entre otros.

El concepto epidemiológico de riesgo es una herramienta útil para la aplicación de programas, tanto para el control de situaciones específicas - como el porte de armas o el consumo de bebidas alcohólicas - como para hacer frente a fenómenos estructurales de mayor complejidad, entre ellos la pobreza y la falta de oportunidades de educación y trabajo.

En Cali es imperativo seguir aplicando medidas dirigidas a disminuir los homicidios y a definir con mayor precisión las acciones más eficaces. Ya se ha comprobado la eficacia del desarme de la población (16), pero esta medida debe aplicarse con mayor constancia y rigor. La realización de campañas sostenidas contra el consumo de bebidas alcohólicas, la investigación acelerada de los móviles que llevan a los asesinatos y la ampliación de la capacidad de los órganos de justicia para reducir significantemente la impunidad, son algunas de las acciones que deben continuarse. Pero aun más allá de todo ello, el Estado y la sociedad tienen que estar conscientes de que la solución de los problemas estructurales, reflejados en la creciente brecha entre pobres y ricos, en la falta de oportunidades de educación y trabajo para los más necesitados, y en la mejoría de los servicios públicos y de la estructura vial para las clases menos favorecidas, producirá un impacto sostenido en la disminución de las actividades delictivas. Combatir la violencia y sus consecuencias es una prioridad social y de salud que no se pueden soslayar.

\section{REFERENCIAS}

1. Secretaría de Salud Municipal de Cali. Perfil epidemiológico de Cali, 1992, 1993, 1994 y salud en cifras, 1995. Cali, Colombia: Oficina de Epidemiología.

2. Concha-Eastman A, Guerrero R, Álvarez A, Cobo G, de Roux G, Alzate A. Programa Desarrollo, Seguridad y Paz (DESEPAZ): estrategias de la Alcaldía de Cali para enfrentar la inseguridad y la violencia. En: Concha-Eastman A, Carrión F, Cobo G, eds. Ciudad y violen- cias en América Latina. Quito: Alcaldía de Cali y Programa de Gestión Urbana de las Naciones Unidas [edición conjunta]; 1994. Pp. 119-153.

3. Espitia VE, Guerrero R, Concha-Eastman A. Vigilancia epidemiológica de los homicidios ocurridos en Cali, enero a diciembre de 1993: la epidemiología aplicada a la administración pública. En: Concha-Eastman A, Carrión F, Cobo G, eds. Ciudad y violencias en América
Latina. Quito: Alcaldía de Cali y Programa de Gestión Urbana de las Naciones Unidas [edición conjunta]; 1994. Pp. 155-166.

4. Guerrero R. Novedoso enfoque de Cali para enfrentar la violencia urbana. La Era Urbana, Violencia Urbana (Banco Mundial) 1993;1(4): 12-13.

5. Guerrero R. Epidemiología de la violencia: el caso de Cali, Colombia. En: Banco Interamericano de Desarrollo. Hacia un enfoque in- 
tegrado del desarrollo: ética, violencia y seguridad ciudadana. Washington, D.C.: BID; febrero de 1996. Pp. 45-68.

6. Espitia VE. Lesiones fatales intencionales y no intencionales en Cali, 1993-1996: un estudio descriptivo. Bogotá: Ministerio de Justicia y del Derecho, Imprenta Nacional de Colombia; 1997.

7. Espitia VE, Guerrero R, Concha A, Sánchez C, Cárdenas V. Patterns of homicide in Cali, Colombia, 1993-94. MMWR 1995;86(4):734-737.

8. De Roux G. Ciudad y violencia en América Latina. En: Concha-Eastman A, Carrión F, Cobo G, eds. Ciudad y violencias en América Latina. Quito: Alcaldía de Cali y Programa de Gestión Urbana de las Naciones Unidas [edición conjunta]; 1994. Pp. 25-45.

9. Instituto Nacional de Medicina Legal y Ciencias Forenses (INMLCF). Comportamiento de las lesiones de causa externa evaluadas por el INMLCF, Colombia 1995, y Comportamiento de las lesiones de causa externa evaluadas por la Regional Bogotá 1995. Bogotá: Grafimpresos; 1996.

10. Atehourtúa A, Guzmán A, Valencia R, Patiño J. La impunidad en Cali. Cali: Editorial XYZ; 1995.

11. Concha-Eastman A, Cruz LF, Carrasquilla G, Cuéllar L, Alzate A, Espinosa R, et al. Patterns of poor health in low-income areas and health sector response in the light of equity: the case of Cali, Colombia. Geneva: World Health Organization; 1997.

12. Concha-Eastman A, Alzate A, Calero D, Correa S, Hernández CA, Muñoz D, et al. Estudio económico, financiero y social de la Secretaría de Salud de Cali. Cali: Departamento de Planeación Municipal; 1996.
13. Sampson RJ, Raudenbush SW, Earls F. Neighborhoods and violent crime: a multilevel study of collective efficacy. Science 1997;277: 918-924.

14. Rutledge R, Messick J. The association of trauma death and alcohol use in a rural state. J Trauma 1992;33(5):737-742.

15. Goodman RA, Istre GR, Jordan FB, Herndon JL, Kelaghan J. Alcohol and fatal injuries in Oklahoma. J Stud Alcohol 1991;52(2):156-161.

16. Villaveces A, Cummings P, Espitia VE, Koepsell TD, McKnight B, Kellerman A. Effect of a ban on carrying firearms on homicide rates in 2 Colombian cities. JAMA 2000;283(9):12051209.

17. Vassar MJ, Kiser KW. Hospitalizations for firearm-related injuries: a population-based study of 9562 patients. JAMA 1996;275:17341739.

18. Concha-Eastman A, Espinosa R. Lesiones violentas no fatales registradas en las comunas 13, 14, 15 y 20 de Cali, 1996. Cali: Universidad del Valle, Centro de Investigaciones Salud y Violencia (CISALVA); 1997.

19. Kellermann AL, Reay DT. Protection or peril? An analysis of firearm-related deaths in the home. New Eng J Med 1986;314:1557-1560.

20. Meehan PJ, O'Carroll PW. Gangs, drugs and homicide in Los Angeles. Am J Dis Child 1992;146(6):693-697.

21. Vanegas G, Bayona JJ. La ciudad y sus violencias: estudios etnográficos en las comunas 13 y 20 de Cali. Universidad del Valle, Centro de Investigaciones Salud y Violencia CISALVA. 1997. Cali: Editorial Feriva; 1998.

22. Smutt M, Miranda L. El fenómeno de las pandillas en EL Salvador. San Salvador: UNICEF, FLACSO; 1998. (Serie Adolescencia No. 2).
23. Kirsch TD, Beaudreau RW, Holder YA, Smith GS. Pediatric injuries presenting to an emergency department in a developing country. Pediatr Emerg Care 1996;12(6):411-415.

24. Singh GK, Yu SM. US childhood mortality, 1950 through 1993: trends and socioeconomic differentials. Am J Public Health 1996;86(4): 505-512.

25. Cruz JM, Trigueros A, González F. El crimen violento en El Salvador: factores sociales y económicos asociados. San Salvador: UCAIUDOP, Banco Mundial; 2000.

26. Franco S. Violence and health in Colombia. Pan Am J Public Health 1997;2(3):170-180.

27. Prothrow-Stith D, Weissman M. Deadly consequences. New York: Harper Collins Publishers; 1991

28. Rosenberg ML. Violence is a public health problem. En: Violence in America: a public health approach. Rosenberg M, Fenley MA, eds. New York, Oxford: Oxford University Press; 1991. Pp. 147-168.

29. Rosenberg ML, O'Carroll PW, Powell KE. Let's be clear: violence is a public health problem. [Commmentaries]. JAMA 1992;267: 3070-1071.

30. Colombia, Ministerio de Salud. Salud y vida en paz: programa del Ministerio de Salud para enfrentar la violencia. Bogotá: Ministerio de Salud; 1997.

31. México, Secretaría de Salud, Asesores en Sistemas de Salud y Centro de Estudios para Prevención de la violencia. La violencia: un problema de salud pública. México, D.F.; Secretaría de Salud; 1999. (Versión en CD Rom).

Manuscrito recibido el 11 de febrero de 2002. Aceptado para publicación, tras revisión, el 15 de julio de 2002.
ABSTRACT

\section{Epidemiology of homicides in Cali, Colombia, 1993-1998: six years of a population-based model}

Objectives. To demonstrate the usefulness of an effective and timely information model, underscore the seriousness of the problem of homicides, and point out the need to apply this type of model as well as comprehensive prevention projects, such as Desarrollo, Seguridad y Paz (DESESPAZ). From 1993 to 1998, 11457 homicides were registered in Cali, Colombia, through an epidemiological surveillance model established under DESESPAZ by the mayor's office in Cali.

Methods. Beginning in January 1993, a work group organized by DESESPAZ reviewed and standardized the variables that different institutions gathered about the victims, their assailants, and the facts surrounding each case, and issued a weekly summary bulletin for the mayor and other local authorities.

Results. Between 1983 and 1994, the homicide rate increased from 23 to 124 per 100000 inhabitants. Subsequently, rates went down in 1995, 1996, and 1997 to 112, 102, and 86,1 per 100 000, respectively, and again rose slightly in 1998 to 88 per 100 000. Even though people of all ages, including children under 5 , have been victims of violence, the most affected group is that of men between the ages of 20 and 34. The ratio of men to women has varied from 14.3:1 to 9.2:1. In terms of numbers, percentages, and rates, low-income groups are the most seriously affected, although the highest-income groups have had rates as high as 160 per 100 000. A firearm was used in over $80 \%$ of homicides, and the crime was most often committed at night and on a weekend. A suspect was identified in only a few cases ( $8 \%$ to $21 \%$ ). The bivariate analysis revealed a positive association with alcohol consumption by the victim, as well as with the use of firearms by the assailant (OR: 3.1; 95\% CI: 2.6 to 3.6). Cases that occurred during a fight between individuals or during group fighting showed an association with the use of a sharp weapon and with alcohol consumption by the victim (OR: 1.9; 95\% CI: 1.4 to 2.6).

Conclusions. A map shows the homicide distribution by neighborhood, and the benefits of a population-based surveillance model are discussed, particularly their usefulness for identifying risk factors and the measures that can be applied to prevent and control this form of violence. 